\title{
Bernoulli Loops in Smoke Rings
}

\author{
Kern E. Kenyon \\ 4632 North Lane, Del Mar, CA, USA
}

Correspondence to: Kern E. Kenyon, kernken@aol.com

Keywords: Smoke Rings, Bernoulli Loops

Received: September 17, $2019 \quad$ Accepted: October 9, $2019 \quad$ Published: October 12, 2019

Copyright $\odot 2019$ by author(s) and Scientific Research Publishing Inc.

This work is licensed under the Creative Commons Attribution International License (CC BY 4.0).

http://creativecommons.org/licenses/by/4.0/

\section{c) (i) Open Access}

\section{ABSTRACT}

Bernoulli's law is applied to the closed streamlines of a smoke ring, and the centrifugal force of the curved flow is balanced by a pressure gradient. Two equations in two unknowns, pressure and velocity, are combined into one equation in one unknown, velocity. Solving the governing equation algebraically produces a radial shear in the velocity such that the speed decreases outward inversely as the radius increases, which is the main result. Measurements are needed to verify the predicted structure of the velocity field.

\section{INTRODUCTION}

After a rough start [1], in most of the intervening two and a half centuries, Bernoulli's law has been under-utilized (and occasionally misused) for helping to understand natural fluid flow phenomena [2]. To expand on the normal concept with open ended streamlines, Bernoulli's law can also be applied to steady flows involving streamlines that are closed loops. One example of that, solid body rotation, has been put into print recently [3]. A second application is to the circular orbits of the fluid particles in a progressive surface gravity wave [4]. Here a third example is explored to see if anything can be learned about the smoke ring circulation. What is predicted below needs to be verified experimentally, without the use of smoke probably, if that is possible to do. This theoretical prediction of the structure of the velocity field has not occurred in the fluid dynamics texts that treat smoke rings as far as I know (e.g. [5]), nor has the method itself appeared in such books.

\section{METHOD}

Pick a circular closed streamline in a smoke ring. What is true for one will be true for all. Pressure along the streamline will be lower than the ambient pressure of the motionless air outside and inside of the streamline, in accordance with Bernoulli's law. Because of the streamline's curvature the flow will experience an outward centrifugal force no matter which way it goes, clockwise or counter-clockwise. For a given radius of curvature, the right speed of flow can be found such that the centrifugal force is balanced by a pressure force. Also the radial thickness of the flow is involved in calculating the pressure force.

Bernoulli's law along a streamline, open or closed, is as always 


$$
p=\text { const }-\frac{1}{2} \rho v^{2}
$$

where $p$ is the pressure, $v$ is the flow speed tangent to the streamline, $\rho$ is the constant fluid density and the constant in Equation (1) is assumed to be the same for all streamlines.

In order to maintain a steady state circulation there must be a balance of forces across the streamlines, i.e. radially (along $r$ ). So the outward centrifugal force must be offset by an equal but opposite inward force. In this fluid flow situation that opposing force can only be a pressure gradient.

$$
\frac{\mathrm{d} p}{\mathrm{~d} r}=\rho \frac{v^{2}}{r}
$$

where $r$ measures distance from the center of the circular orbit.

Two equations in two unknowns can be reduced to one equation in one unknown. For example, take the $r$ derivative of (1) and combine it with (2) to eliminate the pressure with the result

$$
\frac{\mathrm{d} v}{\mathrm{~d} r}=-\frac{v}{r}
$$

Equation (3) has the solution

$$
v=\frac{\text { const }}{r}
$$

To model the smoke ring, the constant in (4) is chosen to be the product of two constants

$$
\text { const }=V_{0} R_{0}
$$

So that when $r=R_{0}$, then $v=V_{0}$, and for $r<R_{0}$ it is assumed that the air is motionless, in the core of the smoke ring.

\section{DISCUSSION}

In the physical/mathematical discussion above there is a framework that is consistent with available smoke ring observations, but specific values for individual variables are not forecast. Is there a core of non-moving air inside the smoke ring, and if so, what is its diameter? Does the rotating layer of flow have a radial shear in it? Answers to these questions are not yet confirmed. Although the smoke exhibits the rotary motion, it obscures the details of features within it. At any rate, the analysis presented may turn out to be an advancement in understanding the physics of the smoke ring hopefully.

If the model of the smoke ring is confirmed, then it will lend strength to other small scale fluid flow applications, such as to the surface gravity wave [6], as well to large scale geophysical situations where the Coriolis force dominates over the centrifugal force, e.g. hurricanes [7]. In other words, the general method will gain creditability.

\section{CONCLUSION}

A physical model of a smoke ring, using Bernoulli's law along the closed streamlines and the force balance method across streamlines, produces a ring of circulating flow that decreases in magnitude radially outward at the rate inversely proportional to the radius from the orbit's center. Experimental data for the velocity shear, which probably do not exist at the present time, are welcomed to compare with the model.

\section{ACKNOWLEDGEMENTS}

Comments by the Reviewer were helpful.

\section{CONFLICTS OF INTEREST}

The author declares no conflicts of interest regarding the publication of this paper. 


\section{REFERENCES}

1. Guillen, M. (1995) Five Equations that Changed the World. Hyperion, New York, 65-118.

2. Kenyon, K.E. (2018) Believing Bernoulli. Natural Science, 10, 142-150. https://doi.org/10.4236/ns.2018.104015

3. Kenyon, K.E. (2019) Bernoulli Loop. European International Journal of Science and Technology, 8, 57-58.

4. Kenyon, K.E. (2019) Bernoulli Loops in Surface Gravity Waves. European International Journal of Science and Technology. (In Press)

5. Faber, T.E. (1995) Fluid Dynamics for Physicists. Cambridge University Press, Cambridge, 157-161. https://doi.org/10.1017/CBO9780511806735

6. Kenyon, K.E. (2013) Depth Decay Rate for Surface Gravity Wave Pressure and Velocity. Natural Science, 5, 44-46. https://doi.org/10.4236/ns.2013.51007

7. Kenyon, K.E. (2019) Hurricane’s Shear. Natural Science. (In Press) https://doi.org/10.4236/ns.2019.119028 